\title{
IV. AUTHORS OF PAPERS READ BEFORE THE AMERICAN MATHEMATICAL SOCIETY, 1891-1904
}

Aley, R. J., (2) IV, 363 ; (2) vI, 185.

Allardice, R. E., (2) vIII, 429; (2) IX, 296, 338, 537; (2) X, 281, 494.

Allen, R. B., (2) x, 486.

Ames, L. D., (2) vili, 368; (2) x, 223.

Baker, A. L., (2) II, 2 ; (2) v, 2, 3.

Ball, R. S., (2) vIII, 185.

Bell, A. T., (2) virI, 319.

Benner, H., (2) IV, 183.

Birkhoff, G. D., (2) Ix, 284; (2) x, 374.

Black, C. W. McG., (2) vII, 290; (2) vIII, 3.

Blake, E. M., III, 130; (2) I, 209 ; (2) III, 2, 2, 200, 353; (2) IV, $2,183,183,363$; (2) V, 2; (2) VI, 179 ; (2) VIII, 429; (2) $\mathrm{x}, 281$.

Blichfeldt, H. F., (2) vI, 373; (2) vII, 374; (2) vIII, 429; (2) IX, 296, 537; (2) X, 281, 494, 494 .

Bliss, G. A., (2) vII, 244; (2) virI, 2; (2) x, 55, 330, 429, 429.

Bôcher, M., I, 231 ; (2) III, 51, 227; (2) IV, 176, 176, 291, 416 ; (2) v, $3,113,326$; (2) VI, $96,179,268$; (2) VII, 3 , 113, 203, 290, 290, 290 ; (2) VIII, $2,3,186,272$; (2) IX, 184, 184, 284; (2) x, 486.

Bolza, O., (2) IV, 3, 183; (2) vI, 185; (2) vII, 244; (2) vIII, 2, $24,319,319 ;(2) \mathrm{Ix}, 74$.

Bouton, C. L., (2) IV, 176; (2) VIII, 199.

Bowden, J., (2) x, 374.

Boyd, J. H., I, 231 ; (2) IV, 183; (2) v, 3.

Brewster, H., (2) Ix, 75.

Bromwich, T. J. I'a., (2) virr, 368, 368; (2) x, 54.

Brown, E. W., (2) II, 276 ; (2) III, 165 ; (2) IV, 88; (2) v, 326, 424 ; (2) VI, 365 ; (2) VII, 374 ; (2) VIII, 24,272 ; (2) IX, 394, $525 ;(2) \mathrm{x}, 374$.

Cajori, F., (2) vI, 2, 365 ; (2) x, 55 .

Campbell, D. F., (2) v, 327; (2) IX, 338.

Campbell, J. E., (2) vI, 179.

Chatburn, G. C., (2) vII, 384.

Chessin, A. S., (2) III, 165; (2) Iv, 88, 176; (2) v, 2, 3, 113, 219, 219,$424 ;(2)$ vI, 2 ; (2) VII, 3 ; (2) IX, $532 ;(2) \mathrm{x}, 56$.

Coble, A. B., (2) vIII, 186 ; (2) Ix, 283.

Cole, F. N., (2) x, 172, 374 .

Collins, J. V., (2) vi, 2 ; (2) vIr, 3; (2) IX, 74; (2) x, 330.

Conant, L. L., (2) v, 3.

Coolidge, J. L., (2) vI, 96 ; (2) vIII, 368.

Cottier, J., (2) III, 257.

Craig, J. E., II, 259.

Craig, T., JI, 147; III, 66.

Crawley, E. S., II, 212.

Curtiss, D. R., (2) vIII, 186, 368; (2) X, 54.

Darwin, G. H., (2) Ix, 283.

Davies, J. E., (2) vI, 2. 
Davis, E. W., II, 35; (2) III, 2, 200, 200; (2) IV, 183; (2) V, 379; (2) VI, 373,374 ; (2) VII, 384 ; (2) IX, 338, 533; (2) $\mathrm{x}, 329$.

Dickson, L. E., (2) IIr, 2, 293; (2) IV, 177, 183, 363; (2) v, 3, 3, $114,223,327,380,380,424$; (2) VI, $3,3,179,185,185,268$, 268,373 ; (2) VII, 244, 244, 289, 374, 384; (2) vIII, 3, 3, 199, $272,319,320$; (2) IX, 74, 74, 75, 184, 184, 283, 296, 338, 338, 526 ; (2) X, 55, 55, 55, 56, 56, 330, 330, 374, 430, 430 .

Dodd, E. L., (2) x, 486 .

Dowling, L. W., (2) IV, 2, 183; (2) vIIr, 3, 199.

Dunkel, O., (2) vIII, 272; (2) Ix, 74.

Durell, F., III, 88.

Echols, W. H., II, 111 ; III, 66 ; (2) I, 237 ; (2) II, 2, 2.

Eddy, H. 'Г., (2) x, 55.

Eiesland, J., (2) IX, 75; (2) X, 54.

Eisenhart, L. P., (2) vII, 203, 290, 290, 374; (2) vIII, 96, 186, 186, 368 ; (2) IX, $75,184,394,526 ;$ (2) x, 172, 374.

Emch, A., (2) III, 200; (2) vi, 2; (2) vII, 243; (2) VIII, 186, 199 ; (2) IX, 75, 338; (2) X, 55 .

Engberg, C. C., (2) IV, 183; (2) V, 223, 380.

Engler, E. A., I, 169; II, 212.

Epsteen, S., (2) VIII, 186, 429; (2) IX, 75, 338, 526, 532, 537; (2) $\mathrm{x}, 55,330,429,430$.

Escott, E. B., (2) x, 429, 429.

Ferguson, O. J., (2) IX, כ̃33.

Field, P., (2) vIrI, 368; (2) Ix, 75, 184, 532.

Fields, J. C., I, 142; (2) IV, 3; (2) vII, 244; (2) vIII, 2, 2, 199.

Findlay, W.g (2) $\mathrm{x}, 374$.

Fine, H. B., II, 76 ; III, 148.

Fiske, T. S., II, 48; III, 148; (2) III, 199.

Fite, W. B., (2) VI, 3. 179; (2) VII, 290 ; (2) vIII, 186, 272 ; (2) $\mathrm{IX}, 74 ;$ (2) X, 223.

Ford, W. B., (2) vII, 202; (2) VIII, 2, 186; (2) IX, 75, 394 ; (2) $\mathrm{X}, 223$.

Frankland, F. W., (2) IV, 3.

Gale, A. S., (2) vI, 365; (2) vIII, 272; (2) IX, 394; (2) X, 172.

Gilman, F., I, 125.

Glover, J. W., (2) III, 353; (2) IV, 183; (2) VIII, 199.

Goodspeed, E. J., (2) IV, 183.

Gordan, P., (2) vi, 96 ; (2) vIr, 3.

Goursat, E., (2) v, 424; (2) x, 172.

Granville, W. A., (2) vII, 374.

Grow, B. E., (2) viI, 3.

Gunderson, C., (2) vIII, 186.

Hadamard, J., (2) vIII, 96.

Hall, T. P., I, 193; (2) I, 160; (2) v, 3, 223; (2) vIII, 199.

Halsted, G. B., (2) I, 2.

Hancock, H., (2) III, 200 ; (2) v, 379; (2) vII, 202; (2) VIII, 185 ; (2) IX, 283, 532 .

Harkness, J., (2) vIr, 4.

Haskell, M. W., (2) I, 97; (2) VIII, 199, 199, 199, 429, (2) x, 281, $282,494,494,495$.

Haskins, C. N., (2) vIII, 3 ; (2) IX, 184; (2) X, 56.

Hathaway, A. S., (2) II, 2; (2) III, 353, 353, 354; (2) IV, 3, 183, $183 ;$ (2) V, 380 ; (2) VII, 243,244 ; (2) IX, 74, 338 .

Hawkes, H. E., (2) IV, 415; (2) VII, 3, 289, 290; (2) IX, 284, 525 ; (2) $\mathrm{x}, 374$.

Hayes, E., (2) IV, 2. 
Hedrick, E. R., (2) v, 327; (2) vIII, 186, 186; (2) IX, 184, 394; (2) $x, 55,329,330$.

Heffter, L., (2) IX, 75.

Henderson, A., (2) Ix, 532, 532.

Henderson, R., (2) II, 52.

Heyl, P. R., (2) Iv, 88, 292.

Hilbert, D., (2) vII, 113.

Hill, G. W., III, 112; (2) I, 55; (2) II, 1, 84, 117; (2) IV, 88; (2) VI, 268 .

Hill, J. E., (2) II, 339 ; (2) III, 2.

Hinton, C. H., (2) IV, 183; (2) vI, 366.

Holgate, T. F., (2) III, 200; (2) IV, 2; (2) v, 2; (2) IX, 75.

Hook, E. A., (2) vII, 243 ; (2) IX, 75 .

Hoskins, L. M., (2) 1x, 296.

Hudson, R. W. H. T., (2) Ix, 283.

Hun, J. G., (2) x, 223.

Huntington, E. V., (2) viII, 96, 271, 272, 272, 368, 368; (2) IX, $74,184,283,283 ;(2) \times, 56,223,223,223,486$

Hutchinson, J. I., (2) III, 2; (2) IV, 3,291 ; (2) v, 2, 423; (2) vI, 179,$268 ;(2)$ vII, $202 ;(2)$ vIII, 2 ; (2) $\mathrm{x}, 55,223$.

Hyde, E. W., (2) III, 2; (2) vII, 374.

Jacoby, H., I, 55; (2) vIII, 185.

Johnson, W. W., I, 231; II, 171, 171; III, 200; (2) I, 209.

Jordan, H. E., (2) x, 330, 330.

Kasner, E., (2) vI, 3; (2) vII, 290, 290, 374, 374; (2) vIII, 3, 96, $186,186,368$; (2) IX, $75,184,283,284,394,526$; (2) $\mathrm{x}, 55$, $55,172,223,374,374,486$.

Kellogg, O. D., (2) x, 223, 486.

Keppel, H. G., (2) viII, 199.

Keyser, C. J., (2) Iv, 177; (2) v, 424; (2) vII, 202; (2) vIII, 368; (2) $\mathrm{Ix}, 75,283 ;(2) \mathrm{x}, 486$.

Kimura, S., (2) IV, 364.

Klein, F., III, 22; (2) III, 92.

Krause, M., (2) vI, 373.

Kuhn, H. W., (2) vi, 268; (2) vII, 3, 113.

Lambert, P. A., (2) II, 2.

La Motte, F. A., (2) vII, 244.

Laves, K., (2) IV, 363 ; (2) v, 379 ; (2) vI, 373 ; (2) viII, 320.

I.ehmer, D. N., (2) III, 200; (2) IV, 183; (2) vIII, 429; (2) IX, 296, $537 ;(2) \mathrm{x}, 282,282,494$.

Lemoine, E., (2) viII, 3.

Lennes, N. J., (2) IX, 533.

Leonard, H. B., (2) x, 429.

Leuschner, A. O., (2) vIII, 430 ; (2) IX, 296.

Ling, G. H., (2) v, 327; (2) vI, 3; (2) IX, 184.

Loewy, A., (2) Ix, 75, 394; (2) x, 55.

Loud, F. H., (2) vI, 365.

Lovett, E. O., (2) III, 293, 353 ; (2) IV, 88, 176, 176, 416, 416; (2) $\mathrm{v}, 3,113,219,326,424$; (2) vI, $96,179,366,366$; (2) VII, $113,203,203,289,290,374,374$; (2) IX, 283; (2) X, 56, $56,486,486$.

Lowell, P., (2) virI, 272.

Lunn, A. C., (2) vIr, 243 ; (2) x, 429, 429.

McClintock, E., I, 125; II, 147; (2) I, 2, 2, 55, 97 ; (2) II, 309; (2) III, 353; (2) IV, 3 ; (2) vII, 202; (2) VIII, 185; (2) IX, 394.

McDonald, J. H., (2) vII, 384, (2) vIII, 430.

McKinney, T. E., (2) vIII, 3. 
McMahon, J., (2) II, 2, 117 ; (2) III, 2; (2) VII, 3.

Macfarlane, A., I, 169, 215; (2) I, 2, 126 ; (2) v, 326 ; (2) vI, 268. Maclày, J., II, 193; (2) IV, 292; (2) VII, 202; (2) vIII, 272.

Manning, H. P., (2) IX, 394.

Manning, W. A., (2) VIII, 430; (2) IX, 296, 537; (2) X, 282.

Martin, A., I, 55; (2) I, 2, 237; (2) II, 2; (2) III, 2; (2) IV, 3; (2) $\mathrm{v}, 3,3$.

Martin, E. N., (2) vI, 3.

Maschke, H., (2) II, 2; (2) III, 200; (2) IV, 3, 183, 364; (2) V, 223,379 ; (2) VI, 185, 373; (2) VIII, 319; (2) IX, 532.

Mason, C. M., (2) x, 56, 374.

Merrill, H. A., (2) $\mathrm{x}, 55$.

Merriman, M., I, 193; (2) IV, 88.

Mertens, F., (2) vir, 384.

Metzler, W. H., (2) IV, 292, 364; (2) VII, 374.

Miller, G. A., (2) III, 199; (2) IV, 3, 176, 183, 292, 416, 416 ; (2) v, $3,219,327$; (2) vI, $3,96,96,178,268,366$; (2) vII, 113 , $290,374,384$; (2) VIII, 3, 96, 186, 199, 430; (2) IX, 284, 296, $394,537,537 ;(2) \mathrm{x}, 172,281,329,494$.

Miller, J. A., (2) vi, 185.

Moore, C. L. E., (2) X, 223.

Moore, E. H. (2) I, 2, 77; (2) II, 2, 233 ; (2) III, 2, 200, 353 ; (2) IV $3,183,364 ;(2)$ v, $3,223,223,379,380 ;$ (2) vI, $3,96,373$, 373 ; (2) vII, 3, 4, 243, 244, 244; (2) vIII, 2, 3, 186, 199, 368; (2) IX, 283.

More, L. T., (2) vi, 3.

Moreno, H. C., (2) viur, 430 ; (2) IX, 537 ; (2) X, 494.

Moritz, R. E., (2) vI, 184, 373; (2) vII, 384, 384.

Morley, F., (2) I, 2, 126, 237; (2) II, 2, 84; (2) III, 165, 353; (2) v, $3,113,424$; (2) vI, $3,96,268$; (2) VII, $3,3,4,202,374$; (2) VIII, $186 ;(2) \mathrm{Ix}, 75,284 ;$ (2) $\mathrm{x}, 56,56,223$.

Moulton, F. R., (2) VI, 2, 185; (2) VII, 3, 244, 384; (2) VIII, 199 ; (2) $\mathrm{Ix}, 74,532$.

Neikirk, L. I., (2) Ix, 526.

Newcomb, S., III, 112; (2) IV, 176.

Newson, H. B., (2) II, 276 ; (2) III, 200, 293, 353; (2) IV, 2, 183, 183 ; (2) V, $223,223,223$; (2) VI, $3,366,373$; (2) vII, 3,243 , 290 ; (2) vIII, 199 ; (2) IX, 75, 338.

Newson, M. W., see Winston, M. F.

Nicholson, J. W., I, 215 ; II, 76.

Noble, C. A., (2) IX, 296.

Nutting, P. G., (2) viII, 430 .

Osgood, W. F., (2) III, 2, 2; (2) IV, 291, 415; (2) v, 3, 3, 326; (2) VII, 3, 4, 202, 374; (2) IX, 283, 283, 394; (2) X, 223.

Peirce, B. O., (2) III, 2; (2) IX, 525; (2) X, 55.

Peirce, C. S., III, 200; (2) I, 77.

Peirce, G., (2) vII, 374.

Peirce, J. M., (2) v, 326 ; (2) x, 486.

Pell, A., (2) vI, 3, 185; (2) VIII, 4; (2) IX, 75.

Penfield, S. L., (2) vII, 114.

Philbrick, P. H., (2) IV, 3.

Pierpont, J., (2) IIT, 51, 257; (2) IV, 292; (2) V, 326, 424; (2) $\mathrm{x}, 486$.

Porter, M. B., (2) III, 257; (2) IV, 292; (2) VI, 268; (2) vII, 3, $289 ;(2)$ viII, 272.

Pringsheim, A., (2) VIII, 2, 2.

Pupin, M. I., I, 101 ; II, 49, 110; (2) II, 233; (2) V, 219, 326, 326 ; (2) VI, $179 ;$ (2) vII, 202. 
Putnam, T. M., (2) vIII, 430; (2) IX, 537.

Quinn, J. J., (2) x, 330.

Rabinovitch, I. E., (2) vIII, 3, 96; (2) IX, 283.

Radelfinger, F. G., (2) viII, 3 .

Rees, J. K., (2) vI, 268.

Reid, L. W., (2) vI, 268.

Richmond, H. W., (2) vI, 2.

Rietz, H. L., (2) vIII, 3, 272; (2) x, 330.

Risteen, A. D., (2) VIII, 368.

Roberts, R. A., (2) I, 97, 189; (2) II, 2, 84.

Roe, E. J., Jr., (2) III, 2, 199; (2) V, 113; (2) vI, 184; (2) vII, 202 ; (2) VIII, 3 ; (2) IX, 75, 283, 283.

Roever, W. H., (2) viII, 272.

Running, T. R., (2) x, 330 .

Rusk, W. J., (2) x, 430.

Safford, F. H., (2) v, 327 ; (2) vII, 243 ; (2) vIII, 368.

Safford, T. H., II, 193.

Saurel, P. L., (2) Iv, 291.

Schlegel, V., (2) I, 2.

Schmiedel, O., (2) vII, 244 ; (2) $\mathrm{x}, 329$.

Schottenfels, I. M., (2) vII, $3,114,244$; (2) viII, $3,96,368$; (2) $\mathrm{Ix}, 184,526,537 ;(2) \mathrm{x}, 172,172,374$.

Schulz, W., (2) v, 219.

Schwatt, I. J., III, 173; (2) x, 54.

Scott, C. A., (2) II, 309; (2) III, 199; (2) IV, 88; (2) v, 424; vI, 96, 96, 365; (2) vIII, 186, 186, 368; (2) IX, 394.

Shaw, J. B., (2) II, 1, 1; (2) III, 2, 200, 353, 353; (2) IV, 2, 183, 363 ; (2) V, 223,380 ; (2) VI, 373 ; (2) vII, 244 ; (2) VIII, 199 ; (2) IX, 74,338 ; (2) $\mathrm{x}, 330,429,429$.

Sisam, C. H., (2) Ix, $526 ;(2) \mathrm{x}, 223,486$.

Skinner, E. B., (2) vII, 244.

Slaught, H. E., (2) VII, 202.

Slocum, S. E., (2) viIr, 96, 186; (2) Ix, 338; (2) x, 56, 430.

Smith, B., (2) x, 172.

Smith, J. A., (2) vI, 374.

Smith, P. F., (2) vII, 3, 114; (2) vIII, 186; (2) x, 223.

Snyder, V., (2) III, 2, 227; (2) IV, 2 ; (2) V, 2, 219, 326; (2) VI, $3,179,179,365$; (2) VII, 3, 202; (2) VIII, 3, 185, 272; (2) Ix, 75,$184 ;(2) \mathrm{x}, 223,486$.

Stabler, E. L., I, 78; (2) V, 3.

Starkweather, G. P., (2) IV, 416; (2) v, 424.

Stecker, H. F., (2) V, 3, 223; (2) VI, 3; (2) vII, 290; (2) vIII, 3, $3,3,96,185,368 ;(2) \mathrm{IX}, 284,526 ;(2) \mathrm{x}, 486$.

Steinmetz, C. P., I, 78; II, 259.

Stolz, O., (2) vIII, 96, 319.

Stone, O., (2) V, 219.

Stringham, I., I, 55; (2) VII, 243; (2) vIII, 430; (2) x, 281, 494.

Stromquist, C. E., (2) Ix, 394.

Strong, W. M., (2) IV, 416; (2) v, 424; (2) vI, 3.

Study, E., III, 66.

Swift, E., (2) x, 172 .

Taber, H., III, 259; (2) I, 126; (2) II, 2, 149; (2) III, 2, 122; (2) v, $326 ;$ (2) vI, $179 ;$ (2) $\mathrm{x}, 374$.

Taylor, W. E., (2) Ix, 283.

Thomson, J. J., (2) IIr, 92.

Timerding, H. E., (2) vII, 3.

Townley, S. D., (2) Ix, 297. 
Townsend, E. J., (2) vII, 244; (2) vIII, 320.

Van der Vries, J. N., (2) x, 55.

Vandiver, H. S., (2) Ix, 284.

Van Vleck, E. B., (2) II, 2; (2) III, 227; (2) IV, 3, 416; (2) V, 327 ; (2) vI, 268 ; (2) vII, 289,374 ; (2) vIII, 2,96 ; (2) IX, $394 ;$ (2) $\mathrm{x}, 119,374$.

Veblen, O., (2) $\mathrm{Ix}, 532$.

Waldo, C. A., (2) vi, 185.

Webster, A. G., (2) IV, 416; (2) V, 327.

Weld, L. G., (2) vIII, 319.

Wernicke, P., (2) IV, 2.

Westfall, J. V., (2) vi, 185.

Westlund, J., (2) V, 3; (2) VII, 384; (2) VIII, 199, 199; (2) IX, 75; (2) $\mathrm{x}, 55,330$.

White, H. S., (2) I, 2; (2) II, 149; (2) III, 2, 200, 200, 353; (2) IV, 3 , 183, 292, 416; (2) v, 223 ; (2) vI, 2, 185; (2) vIII, 199 ; (2) IX, 75, 338, 532; (2) X, 119, 330 .

Whitney, A W., (2) vIII, 430; (2) x, 494 .

Whittaker, E. T., (2) x, 55.

Whittemore, J. K., (2) IV, 415; (2) vI, 96 ; (2) vII, 203; VIII, 96.

Wilczynski, E. J., (2) v, 219; (2) vI, 184, 365; (2) vII, 114, 374 ; (2) vIII, $96,272,430$; (2) IX, 297, 537; (2) X, 223, 281, 281, 486.

Williams, E. C., II, 35, 48.

Willson, R. W., (2) III, 2.

Wilson, E. B., (2) v, 424; (2) vI, 96; (2) Ix, 283, 526; (2) x, $56,223$.

Wilson, R. E., (2) Ix, 338.

Winston, M. F., (2) III, 200.

Wood, R. G., (2) vir, 202; (2) vIII, 368.

Woods, F. S., (2) x, 119.

Woodward, R. S., (2) I, 2, 209 ; (2) II, 52; (2) IV, 88, 88, 176, 292 ; (2) V, 113 ; (2) VI, 179,366 ; (2) IX, 526 ; (2) X, 56, 56.

Young, A. E., (2) x, 429.

Young, J. W., (2) vIII, 186, 272; (2) IX, 74, 283.

Young, J. W. A., (2) v, 223, 380; (2) VII, 4.

Young, W. H., (2) x, 55. 